\title{
Sociodemographic Comparison in Patients with Subjective and Objective Clinical Findings of Temporomandibular Dysfunctions
}

Sevgi Senera

Faruk Akgunlua

\begin{abstract}
Objectives: To investigate the differences in healthy persons and patients with myofacial pain without limitation, objective signs of temporomandibular dysfunctions (TMDs), and both subjective and objective features with respect to age, gender, effects of TMDs on daily life, economic condition, smoking habit, marital status, and education.

Methods: Sociodemographic information of 296 persons (212 females and 84 males) was evaluated. Subjects were categorized into 4 groups according to clinical findings: no TMD symptoms (control, C); myofacial pain without limitation (MP); objective signs (PWOS); or both subjective and objective features (MP/PWOS).

Results: The C, MP, PWOS, and MP/PWOS groups comprised 64 (22\%), 66 (22\%), 68 (23\%), and $98(33 \%)$ subjects. PWOS and MP/PWOS differed significantly between the 15-30 and 30-45 year age groups. Although patients with PWOS were significantly higher in the 15-30 year age group, patients with MP/PWOS were significantly higher in the 30-45 year age group. A significant difference was observed with respect to gender in all groups except the control group. The effects of TMD symptoms on daily life differed statistically among the groups.

Conclusions: Female gender is a risk factor for TMDs. Females are more vulnerable to subjective and objective signs than males. During general dental examinations, females should be checked for TMD symptoms. PWOS manifest before MP. Moreover, mechanical derangements can cause myofacial pain. MP can mostly affect daily life, but PWOS tend to be prohibitive. (Eur J Dent 2011;5:380-386)
\end{abstract}

Key words: TMD; Age; Gender; Myofacial pain.

\footnotetext{
a Selcuk University, Faculty of Dentistry, Department of Oral Radiology, Konya, Turkey.

Corresponding author: Sevgi Sener Selcuk University, Faculty of Dentistry, Department of Oral Radiology, Konya, Turkey. Phone: +90 3322231209

Fax: +903322410062

E-mail:sevgi_senerahotmail.com
}

\section{INTRODUCTION}

"Temporomandibular dysfunctions" (TMDs) is an umbrella term referring to many clinical problems that involve preauricular and temporomandibular joint (TMJ) areas. The patients suffer from muscle and/or joint pain and/or joint dysfunctions. ${ }^{1-3}$ The differences in sociodemographic 
characteristics among healthy persons; patients with subjective features such as muscle pain; and patients with objective dysfunctions, such as clicking sounds, mandibular deviation, deflection, and limitation of mouth opening, may be important. These differences can guide the diagnosis, prevention, and treatment of TMDs.

A systematic review of potential risk factors stated that the effects of both age and gender should be taken into account. 4,5 Additionally, the effects of social factors, such as marital status, smoking, and education, as possible risk factors for the development of TMD have also been investigated. ${ }^{6-12}$ However, a consensus has not yet been reached as to whether the sociodemographic factors are involved in the development of TMDs. ${ }^{5,7}$

The aim of this study was to investigate the differences among healthy persons; patients with myofacial pain without limitation; patients with objective signs such as clicking, deviation, deflection, and limitation; and patients with both MP and objective features with respect to age, gender, the effects of TMDs on daily life, economic condition, smoking, marital status, and education.

\section{MATERIALS AND METHODS}

In a 3 month period, 296 patients $(212$ females and 84 males) with orofacial pain were referred to the Oral Diagnosis Clinic. The mean age of the patients was 32.01 years (range, 15-45 years).

The subjects were categorized into 4 groups $^{14}$ according to the results of the clinical examination: no signs or symptoms (control, C); myofacial pain without limitation (MP); objective features such as clicking, deviation, deflection, and limitation (hard-end-feel) (PWOS); and both MP and objective signs (MP/PWOS).

The $\mathrm{C}$ group consisted of patients from the clinic with myofacial pain caused by conditions other than TMDs. The myofacial pain in the control subjects was caused by different conditions such as tooth ache, sialolithiasis, impacted third molars, trigeminal neuralgia, gingival or periodontal infection, and mucosal ulcers. The differential diagnosis was obtained by detailed clinical and radiological examinations.

MP was considered as a subjective symptom of TMDs. Pain in the jaw muscles or in the joint in front of the ear or inside the ear lother than infection) within the past 6 months was recorded as MP. ${ }^{6}$ Clicking sound, deviation, deflection, and limitation (hard-end-feel) on mouth opening were recorded as the objective features of TMDs. ${ }^{12,13}$ In the patients of the MP/PWOS group, both subjective and objective symptoms were present.

Sociodemographic information was obtained by interview. Gender, age, economic condition, education level, marital status of the patient, and the effects of signs and/or symptoms of TMDs on daily life of the patient were recorded.

The participants were categorized into the following 4 groups according to the economic condition, i.e., according to the total monthly income (contribution of all family members was considered): (1) 500-1000 Turkish Lira (TL); (2) 1000-2500 TL; (3) 2500-4000 TL; and (4) $4000 \mathrm{TL}$ and above.

The subjects were categorized into the following 3 groups according to the marital status: (1) single (not married); (2) married; and (3) divorced.

According to the smoking (cigarette, cigar, and pipe) habits, the subjects were classified into the following 2 groups: smoking and nonsmoking. The frequency and amount of smoking were not considered.

The education level of persons was recorded as the total time in years spent on education Ifor example, graduation from elementary school was recorded as 5 years and graduation from secondary school was recorded as 8 years).

The effects of the signs and symptoms on the daily life of persons were judged by asking the patients to grade the condition as having no effects, having some effects, or being prohibitive. An index was not used in this study to determine the effects of signs and symptoms. The effects of TMDs were determined by clinical anamnesis of the patients.

The data were analyzed by SPSS 13.0 (SPSS Inc., III, USA). The chi-square test (for gender, age, marital status, smoking, education, and effects on daily life) and one-way analysis of variance (ANOVA) (economic condition) were used for statistical assessment.

\section{RESULTS}

The C, MP, PWOS, and MP/PWOS groups consisted of $64(22 \%), 66(22 \%), 68(23 \%)$, and 98 (33\%) participants, respectively (Table 1). The mean ages of subjects in the C, MP, PWOS, and MP/PWOS groups were 21.5 (15-40), 31 (17-45), 26 (16-45), and 31 (17-45) years, respectively. In 
total, there were 164 patients with myofacial pain (subjective symptom) (in MP + MP/PWOS) and 166 patients with objective findings (in PWOS + MP/ PWOS). Of the 166 patients with objective findings, clicking, deviation, clicking and deviation, deflection, limitation, and deflection and limitation were observed in 75 (45\%), 11 (7\%), 4 (4\%), 44 (27\%), 12 $(7 \%)$, and $20(10 \%)$ patients, respectively.

A significant difference was found between the 15-30 year age group and the 30-45 year age group ( $P=0.00$ ) (ANOVA). Although objective signs were the most prevalent in the 15-30 year age groups, MP/PWOS were the most prevalent in the 30-45 year age group (Table 1). A significant difference was found between MP and PWOS in the 15-30 year age group. In the 30-45 year age group, a significant difference was found between $C$ and PWOS, $C$ and MP/PWOS, MP and PWOS, MP and
MP/PWOS, and PWOS and MP/PWOS. The number of patients with MP was equal in the 15-30 and 3045 year age groups. The number of patients with objective signs was significantly higher in the 1530 year age group, while the number of patients with MP/PWOS was significantly higher in the 30-45 year age group. MP/PWOS were commonly observed in the female patients, while PWOS were the most common symptoms in the male patients. The effects on the daily life differed significantly among the groups ( $P=0.00$ ) (Chi-square test) (Tables 2 and 3). Most patients with MP/PWOS stated that the conditions affected their daily life. PWOS were commonly prohibitive on the daily life of persons. The groups did not show any difference with respect to the education level, smoking, marital status, and economic conditions (P>.05) (Chisquare test) (Tables 4-7).

Table 1. Number and percentage (in parentheses) of controls and subjects with MP, PWOS, and MP/PWOS in the different age groups.

\begin{tabular}{lccc}
\hline Groups & $15-30$ years & $30-45$ years & Total \\
\hline C & $34(56)$ & $30(44)$ & $64(22)$ \\
MP & $33(50)$ & $33(50)$ & $66(22)$ \\
PWOS & $54(80)$ & $14(20)$ & $68(23)$ \\
MP/PWOS & $38(39)$ & $60(61)$ & $98(33)$ \\
Total & $159(54)$ & $137(46)$ & 296 \\
\hline
\end{tabular}

Table 2. Gender distribution of subjects across the groups expressed as number and percentage in parentheses.

\begin{tabular}{lccc}
\hline Groups & Female & Male & Total \\
\hline C & $32(50)$ & $32(50)$ & $64(22)$ \\
MP & $56(85)$ & $10(15)$ & $66(22)$ \\
PWOS & $40(59)$ & $28(41)$ & $68(23)$ \\
MP/PWOS & $84(86)$ & $14(16)$ & $98(33)$ \\
Total & $212(72)$ & $84(28)$ & 296 \\
\hline
\end{tabular}

Table 3. Effects of TMDs on daily life expressed as number of subjects and percentage in parentheses in the different groups.

\begin{tabular}{lcccc}
\hline & No effects & Some effects & Prohibitive & Total \\
\hline C & $64(53)$ & 0 & 0 & $64(22)$ \\
MP & $18(15)$ & $42(32)$ & $6(14)$ & $66(22)$ \\
PWOS & $22(18)$ & $26(29)$ & $20(48)$ & $68(23)$ \\
MP/PWOS & $16(13)$ & $66(49)$ & $16(38)$ & $98(33)$ \\
Total & $120(41)$ & $132(45)$ & $42(14)$ & 296 \\
\hline
\end{tabular}

Table 4. Marital status of the subjects across the different groups expressed as number of subjects and percentage in parentheses.

\begin{tabular}{|c|c|c|c|c|}
\hline Groups & Single & Married & Divorced & Total \\
\hline C & 38 (27) & $26(17)$ & 0 & $64(22)$ \\
\hline MP & $22(15)$ & $44(29)$ & 0 & $66(22)$ \\
\hline PWOS & 40 (28) & $26(17)$ & $1(50)$ & 68 (23) \\
\hline MP/PWOS & $42(30)$ & 56 (37) & $1(50)$ & 98 (33) \\
\hline Total & 142 (48) & 152 (51) & $2(0.6)$ & 296 \\
\hline
\end{tabular}




\section{DISCUSSION}

In TMDs, treatment is directed to the elimination of the pain and/or dysfunction such as limitation. Medicines such as analgesics and anti-inflammatory drugs, psychological consultation, different intraoral appliances and splints, and arthrocentesis (hydraulic distension) have been used for elimination of the etiologic factor(s) of the complaints. ${ }^{1,2}$ TMJ imaging is necessary when the symptoms persist or worsen during the treatment. For cases with only clicking and/or deviation, TMJ imaging is not necessary. ${ }^{15}$ Magnetic resonance imaging (MRI) is used for determination of the position and signal intensity of the articular disc, effusion, and adhesions. In addition, bone changes can be imaged without radiation. However, MRI is not necessary at the beginning of the treatment. MRI facilities are not available in every medical or dental center; further, the procedure is time consuming and expensive. Computed tomography and arthrography are invasive and unnecessary methods for the beginning of the assessment. Therefore in the current study, the subjects were categorized only according to the clinical findings. In addition, many studies have revealed a significant correlation between clinical and MRI findings. ${ }^{16-18}$
"TMDs" is an umbrella term referring to many clinical problems that involve preauricular and TMJ areas. ${ }^{1,2}$ The subgroups can show different sociodemographic features. Thus, these variations can be effective in the prevention and treatment of TMDs. Therefore, we evaluated the sociodemographic differences between the patients with subjective and objective clinical findings of TMDs.

TMDs have been reported to primarily affect young and middle-aged adults, and they are approximately twice more common in women than in men in the general population. ${ }^{5}$ The study of Suvinen et $\mathrm{al}^{14}$ did not reveal any statistical difference in the age of patients among the 3 groups. ${ }^{14}$ Reißmann et $\mathrm{al}^{7}$ and Huang et $\mathrm{al}^{6}$ stated that the mean age of subjects did not differ significantly among the different groups. In the study of Huang et $\mathrm{al}^{6}$, the mean ages of controls, patients with myofacial pain, patients with arthralgia, and patients with myofacial pain/arthralgia were 40, 39, 44 , and 38 years, respectively. Suvinen et al ${ }^{14}$ stated that the mean ages of patients with complex, intermediate, and simple cases were 39,43 , and 45 years, respectively. In this study, the mean ages were 21.5 (15-40), 31 (17-45), 26 (16-45), and 31 (17-45) years for the C, MP, PWOS, and MP/PWOS

Table 5. Education level of the subjects across the groups expressed as number of subjects and percentage in parentheses.

\begin{tabular}{|c|c|c|c|c|c|}
\hline Groups & $0-5$ years & $5-10$ years & $10-15$ years & 15 years and above & Total \\
\hline C & 0 & 2 (3) & $14(20)$ & $48(31)$ & $64(22)$ \\
\hline MP & $2(100)$ & $18(26)$ & $18(26)$ & $28(18)$ & $66(22)$ \\
\hline PWOS & 0 & $16(24)$ & $16(23)$ & $36(23)$ & $68(23)$ \\
\hline MP/PWOS & 0 & $32(47)$ & $22(31)$ & $44(28)$ & 98 (33) \\
\hline Total & $2(0.6)$ & $68(23)$ & $70(24)$ & $156(53)$ & 296 \\
\hline
\end{tabular}

Table 6. Financial condition of the subjects according to the groups.

\begin{tabular}{|c|c|c|c|c|c|}
\hline Groups & $500-1000 \mathrm{TL}$ & $1000-2500 \mathrm{TL}$ & $2500-4000 \mathrm{TL}$ & 4000 and over TL & Total \\
\hline C & $6(14)$ & $32(20)$ & $16(26)$ & 10 (39) & $64(22)$ \\
\hline MP & $10(23)$ & 44 (27) & $10(16)$ & 2 (8) & $66(22)$ \\
\hline PWOS & $16(36)$ & $28(17)$ & $16(26)$ & $8(31)$ & $68(23)$ \\
\hline MP/PWOS & $6(14)$ & 60 (37) & $20(32)$ & $6(23)$ & 98 (33) \\
\hline Total & $44(15)$ & $164(55)$ & $62(21)$ & 26 [9] & 296 \\
\hline
\end{tabular}

Table 7. Presentation of the correlation between the smoking habits and the groups

\begin{tabular}{lccc}
\hline Groups & Smoking & Nonsmoking & Total \\
\hline C & $40(18)$ & $24(35)$ & $64(22)$ \\
MP & $56(25)$ & $10(15)$ & $66(22)$ \\
PWOS & $56(25)$ & $12(18)$ & $68(23)$ \\
MP/PWOS & $76(33)$ & $22(32)$ & $98(33)$ \\
Total & $228(77)$ & $68(23)$ & 296
\end{tabular}


groups, respectively. The difference can result from the mean age of patients and categorization of patients according to the clinical findings in previous studies. According to these findings, it may be concluded that the TMD process begins with disc displacement. Mechanical problems such as disc displacement can result in myofacial pain and muscle fatigue. For instance, mechanical incoordination between the disc and the condyle can act as a trigger factor in the development of myofacial pain. However, there are many possible etiological factors in the development of TMDs and these factors can contribute to the process synergistically. Therefore, in all cases with disc displacement, myofacial pain may not develop. If the disc displacement is identified at an early age and the possible contributors can be eliminated, myofacial pain may not develop. The mean age of our patients is consistent with the age generally reported in the literature ${ }^{6}$ but the mean ages of subjects in other previous studies were higher. ${ }^{7,10}$ Therefore, TMDs are seen in young and middleage adults. Accordingly, younger persons should be examined, and for patients showing the signs or symptoms, preventive actions should be taken and the patients should be informed about TMDs in dentistry.

In the present study, the percentages of subjective and objective findings were higher in females than in males. In particular, MP and MP/ PWOS were significantly higher in females. This finding is in accordance with those in the literature. ${ }^{6}$ Muscular symptoms can be observed more frequently in females because they are subjected to other possible etiologic factors such as depression, emotional stress, and bruxism. In females in particular, psychological consultation should be added to the treatment plan for TMDs.

This study revealed that subjective symptoms affected daily life and objective findings of the dysfunctions had a prohibitive effect on the daily life of the patients. Social and psychological conditions and efficiency of working, eating, and talking can be affected by TMDs. It can be considered that in anterior disc displacement without reduction, movement limitation can be a prohibitive complaint for the patients. Therefore, prevention and treatment of TMDs can enhance the life quality of patients, especially those with disc displacement. These findings are not in agreement with those in the literature. ${ }^{19}$ It has been reported that subjects with TMJ pain had the lowest level of disability, while subjects with pain in and around the temples (myogenous pain) received the highest amount of medication for their complaints. Bush and Harkins $^{19}$ reported higher pain-related limitations in daily life activities in patients with myogenous pain than in patients with arthrogenous pain. Miller et $\mathrm{al}^{20}$ found that TMD patients with high mandibular opening index had more severe signs and symptoms of TMD than patients with a low index. However, mandibular opening can be restricted with both muscle spasm and intra-articular hampers or the muscle pain can cause limitation secondarily. Bush and Harkins ${ }^{19}$ evaluated the effects of TMD signs and symptoms on daily life by using the pain disability index. In that study, only the effects of pain were assessed and those of objective signs were not assessed. We did not use any index to evaluate the effects of TMD on daily life; we simply asked patients whether the present complaints affected or hindered their daily life activities such as talking, eating, working, etc. Therefore, our question was more general and was not directed only to pain. In our subjects, objective signs were found to have prohibitive effects on daily life activities. This finding may result from limitation cases. The discrepancies between our findings and previous findings can result from the methods used, and probably the perception and referring of the symptoms of TMDs can vary according to the nationality.

A previous study revealed that patients diagnosed with myofacial pain and a combination of myofacial and joint pain had significantly higher levels of financial stress than the controls. ${ }^{9} \mathrm{Fa}-$ cial pain was also found to be higher in the urban population. ${ }^{21}$ In this study, the economic condition of the subjects did not differ between the controls and the groups. Thus, TMDs are not prevented by enhancing the economic conditions or this factor does not affect the development of TMDs on its own. The economic condition of a person can be good, but that person can have financial stress. The difference can result from classification of subjects. Furthermore, the proportion of subjects in the different economic condition groups differed in the present study. Therefore, absolute results cannot be obtained from the findings of this study. In this study, marital status was not found to be 
correlated with the presence of TMDs. This finding confirmed those in the literature, ${ }^{2,6}$ but problems in marriage can cause the symptoms of TMDs owing to psychological stress. It is not important whether to be single or married; the view point of each person can be different to be marriage and to be single.

The smoking habit did not differ between the controls and the subjects of the other groups in the present study. Our findings agreed with those of other studies. ${ }^{11,12}$ The signs and symptoms of TMDs do not trigger the consumption of tobacco according to the finding of this study. However, the proportion of smoking subjects was significantly lower $(23 \%)$ than that of nonsmoking subjects in our study. Therefore, definite results about the correlation between the smoking habit and TMDs cannot be obtained from the findings of this study.

The education level did not differ significantly among the groups in this study. This finding is in agreement with those in the literature. 1,2 Therefore, increase in the level of education cannot prevent the development of signs and symptoms of TMD; further, the incidence of TMD is not higher in patients with a low education level. However, the percentage of persons with a high level of education (15 years and above) was significantly higher in our study. Thus, to obtain an absolute result, the number of subjects in each education group should be equalized.

\section{CONCLUSIONS}

This study revealed that age and especially gender are risk factors for TMDs. Daily activities of TMD patients are limited especially in patients with objective symptoms, and TMDs can decrease the quality of life of persons. Females are more vulnerable to all forms of TMDs. Female patients in the Turkish population should be examined for the signs and symptoms of TMDs during general dental examination. The objective features of TMDs occur earlier in life than MP. It can be concluded that the progression of TMDs can begin with mechanical derangement and can lead to myofacial pain. In cases with anterior disc displacement with/without reduction, caution should be taken to prevent myofacial pain.

\section{REFERENCES}

1. Bell WE. Orofacial pains: classification, diagnosis, management. $4^{\text {th }}$ edition, Year Book Medical Publishers, Chicago 1989: p.101.

2. Okeson J, Orofacial pain. $5^{\text {th }}$ edition, Quentissence Publishing, Illionis, 1996.

3. Shmitter M, Kress B, Ohlmann B, Henningsen P, Rammalsberg P. Psychosocial behavior and health care utilization in patients suffering from temporomandibular disorders diagnosed on the basis of clinical findings and MRI examination. Eur J Pain 2005;9:243-250.

4. Drangsholt MT, Leresche L, Von Korff F, Trulove E, Sommers E. Temporomandibular disorders pain. In: The epidemiology of pain. Crombie IK. Seattle: IEASP Press 1989.

5. Leresche L. Epidemiology of temporomandibular disorders: implications for investigation of etiological factors. Crit Rev Oral Biol Med 1997;8:291-305.

6. Huang GJ, LeResche L, Critchlow CW, Martin MD, Drangsholt MT. Risk factors for diagnostic subgroups of painful temporomandibular disorders. $J$ Dent Res 2002;81:284-288.

7. Reißmann DR, Jhon MT, Schierz O, Wassel RW. Functional and psychosocial impact related to specific temporomandibular disorder diagnoses. J Dent 2007;35:643-650.

8. Tesch RS, Denardin OVP, Baptista CA, Dias FL. Depression level in chronic orofacial pain patients: a pilot study. J Oral Rehabil 2004;31:926-932.

9. Akhter R, Hassan NM, Aida J, Kanehira T, Zaman KU, Morita M. Association between experience of stressful life events and muscle-related temporomandibular disorders in patients seeking free treatment in a dental hospital. Eur J Med Res 2007;12:535-540.

10. Goddard G, Karibe H. TMD prevalence in rural and urban Native American populations. Cranio 2002;20:125-128.

11. Bernhardt O, Gesch D, Schwahn C, Mack F, Meyer G, John $U$, Kocher T. Risk factors for headache, including TMD signs and symptoms, and their impact on quality of life. Results of the Study of Health in Pomerania (SHIP). Quintessence Int 2005;36:55-64

12. Wänman A. Temporomandibular disorders among smokers and nonsmokers: a longitudinal cohort study. J Orofac Pain 2005; 19:209-217.

13. Dworkin SF, LeResche L. Research diagnostic criteria for temporomandibular disorders: review, criteria, examination and spesifications, critique. J Craniomandib Disord 1992;6:301.

14. Suvinen TI, Reade PC, Hanes KR, Könönen M. Temporomandibular disorder subtypes according to the self-reported physical and psychosocial variables in female patients: a reevaluation. J Oral Rehabil 2005;32:166-173. 
15. White SC, Pharoah MJ. Oral Radiology: Principles and Interpretation. 5th edition, Mosby, St. Louis Missouri 2004; p. 493-528.

16. Usumez S, Oz F, Guray E. Comparison of clinical and magnetic resonance imaging diagnosis in patient with TMD history. J Oral Rehabil 2004;31:52-56.

17. Yatani H, Sonoyoma M, Kuboki T, Matsuka Y, Orisini MG, Yamashita A. The validity of clinical examination for diagnosing anterior disc displacement with reduction. Oral Surg Oral Med Oral Pathol Oral Radiol Endod 1998;85:647-653.

18. Aoyama S, Kino K, Amagasa T, Sakamoto I, Omura K, Honde $E$, et al. Clinical and magnetic resonance imaging study of unilateral sideways disc displacements of the temporomandibular joint. J Med Dent Sci 2002;49:89-94.

19. Bush FM, Harkins SW. Pain-related limitation in activities of daily living in patients with chronic orofacial pain: psychometric properties of a disability index. J Orofac Pain 1995;9:57-63.

20. Miller VJ, Karic VV, Myers SL. Differences in initial symptom scores between myogenous TMD patients with high and low temporomandibular opening index. Cranio 2006;24:25-28.

21. Glaros AG, Willams K, Lausten L. The role of parafunctions, emotions and stress in predicting facial pain. Am Dent Assoc $2005 ; 136: 451-458$. 